CERIAS Tech Report 2004-118

Modeling Search in Group Decision Support Systems

by J Rees, G Koehler

Center for Education and Research

Information Assurance and Security

Purdue University, West Lafayette, IN 47907-2086 


\title{
Modeling Search in Group Decision Support Systems
}

\author{
Jackie Rees and Gary J. Koehler
}

\begin{abstract}
Groups using group decision support systems (GDSS) to address particular tasks can be viewed as performing a search. Such tasks involve arriving at a solution or decision within the context of a complex search space, warranting the use of computerized decision support tools. The type of search undertaken by the groups appears to be a form of adaptive, rather than enumerative, search. Recently, efforts have been made to incorporate this adaptation into an analytical model of GDSS usage. One possible method for incorporating adaptation into an analytical model is to use an evolutionary algorithm, such as a genetic algorithm (GA), as an analogy for the group problem-solving process. In this paper, a test is made to determine whether GDSS behaves similarly to a GA process utilizing rank selection, uniform crossover, and uniform mutation operators. A Markov model for GAs is used to make this determination. Using GDSS experimental data, the best-fit transition probabilities are estimated and various hypotheses regarding the relation of GA parameters to GDSS functionality are proposed and tested. Implications for researchers in both GAs and group decision support systems are discussed.
\end{abstract}

Index Terms-Genetic algorithms, group decision support systems, Markov processes.

\section{INTRODUCTION}

G ROUPS of individuals meeting to solve particular problems can be viewed as searching for an optimal or nearoptimal solution within a solution space [1]. This solution space is likely to be highly complex; otherwise, the best solution could be located by an individual, rather than requiring the effort of a group of individuals. Group support systems, in particular, group decision support systems (GDSS), have been used to assist groups in their problem-solving efforts.

GDSS are designed to support group decision-making through specialized software, hardware, and decision support tools. DeSanctis and Gallupe in [2] defined GDSS as a combination of computer, communications, and decision technologies working in tandem to provide support for problem identification, formulation, and solution generation during group meetings. For our purposes, we do not restrict the term GDSS to the traditional decision room and facilitator.

Using the above definition, GDSS could be construed as a tool for facilitating the search for better solutions within the defined solution space. Not only does GDSS provide decision support tools for modeling and solving problems, but also tools for reducing the "process losses" and emphasizing the "process gains" assumed to be present in typical GDSS usage [3]. Within

Manuscript received September 1, 1999; revised October 15, 2003. This paper was recommended by J. Chen.

J. Rees is with the Krannert Graduate School of Management, Purdue University, West Lafayette, IN 47907-2056 USA (e-mail: jrees@ mgmt.purdue.edu).

G. J. Koehler is with the Decision and Information Sciences Department, Warrington College of Business, University of Florida, Gainesville, FL 32601 USA (e-mail: koehler@ufl.edu).

Digital Object Identifier 10.1109/TSMCC.2004.829307 this context, GDSS is a tool to improve the efficiency of the group's search through the solution space.

A phenomenon of interest to GDSS researchers is the patterns of search behavior present in group decision-making. Once the patterns of search are better understood, GDSS variables and the relationships between search patterns and those variables can be re-examined, with the eventual goal of making GDSS as efficient and effective as possible. Examples of GDSS variables include proximity (face-to-face or geographically dispersed), incentive structure, degree of anonymity, leadership presence, group size, and composition, etc.

The pattern or type of search typical in GDSS use is most likely not a random search; otherwise, GDSS would have no beneficial effects over certain groups. However, the search is most likely not deterministic in an algorithmic sense. Hirokawa and Johnson proposed that the group decision-making process is an evolutionary one [4]. There exists a class of algorithms that behave like evolutionary processes, so-called evolutionary algorithms. The genetic algorithm (GA) is a special type of evolutionary algorithm particularly suitable for modeling the search activities of groups using GDSS.

This paper describes and improves upon an earlier analytical model for GDSS search using a GA as the basis of the model [5]. The model is improved by incorporating more sophisticated implementations of the selection and recombination operators that should better capture the dynamics of a GDSS process. We test this model using experimental data and present the results of tests of hypotheses linking GA parameters to GDSS variables, specifically geographic proximity and incentive structure. Earlier studies [5], [6] using a simpler GA model focused on geographic proximity and leadership variables. These results are further underscored by the overall robustness of the generalized GA approach presented in Rees and Koehler [7].

The remainder of the paper is organized as follows: Section II briefly presents existing analytical models for GDSS as well as the mechanics and characteristics of the GA. Section III presents the model for GDSS search and research questions based on the model and are put forth in Section IV. Section V discusses the experimental data used to test the research questions and the results that are contained in Section VI. Finally, the conclusions and future research are presented in Section VII.

\section{BACKGROUND}

A brief overview of two previous analytical models for GDSS processes is presented. The relative strengths and weaknesses of the models are highlighted along with the justification for why another analytical model would be useful for both researchers and practitioners. We also present in this section a brief background on GAs as they are used in this particular study. The mechanics and underlying theory of the GA as pertaining to this re- 
search and justification for why GAs are suitable as a modeling tool are discussed.

\section{A. Analytical Models for GDSS}

Valacich and Dennis in [8] presented a simple mathematical model of electronic brainstorming using GDSS. Their model presents GDSS brainstorming as the ideas generated by a group of individuals, each working alone, account for process losses and process gains [3]. In other words, “... group performance is a function of individual performance minus process losses plus process gains" [8, p. 64]. Their model was one of the earliest models to provide analytical insight into a particular GDSS process. The shortcoming of the model is that it can provide no insight into the expected behavior of the system, where the system is composed of the group members, the environment, the task under consideration, the reward (internal or external) tied to decision quality, and the final decision itself.

Perhaps the most closely related GDSS research to this particular research project is the economic analysis of GDSS [9]. This work was preceded by research on distributed GDSS [10], [11] where brainstorming and other GDSS activities were closely examined. One of the important features of their model is that it considers GDSS use by groups to be in the format of a search problem with a very large search space. According to their model, every feasible solution has a payoff, which must be balanced with the cost of performing the search. Another aspect of interest in their model is the discussion of a "trigger phenomenon" [9]. This is the case when an original idea "triggers" a new line of reasoning or discussion. The model also addressed the probability of finding a solution, the expected net benefit of finding a particular solution, stopping criteria, and the marginal value of group size [9]. The one drawback to their model is the difficulty in understanding the underlying processes in GDSS use. For example, the trigger phenomenon is described but is not easily operationalized. However, this phenomenon is remarkably similar to the mutation operation of the GA, discussed below.

\section{B. GAs}

In order to provide the type of analysis that would prove useful to researchers and practitioners, the appropriate tool must be selected as the basis of such a model. We propose that the GA as described in [12] is such a tool.

GAs are general-purpose search algorithms driven by the basic principles of Darwinian natural selection and evolution. Search is performed from a population of agents, rather than from a single one. Such agents, called strings, points, or chromosomes, explore a space using three basic operations. Selection or reproduction is the operator that tends to take the "fittest" members of the population for use in generating the next generation. Crossover combines genetic material between selected members of the population. Finally, mutation adds diversity back into a population. This process is illustrated in Fig. 1.

The GA operations proceed as follows. First, strings are evaluated according to a given fitness function. This evaluation, or objective value, influences the proportion of the string in the next time series, or generation. Fitter strings generally have a

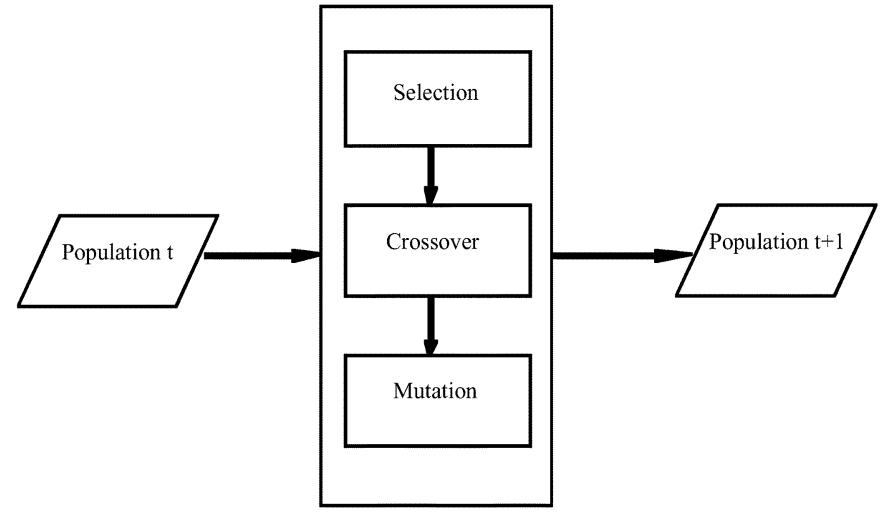

Fig. 1. Simple GA process.

TABLE I

SIMPLE GA

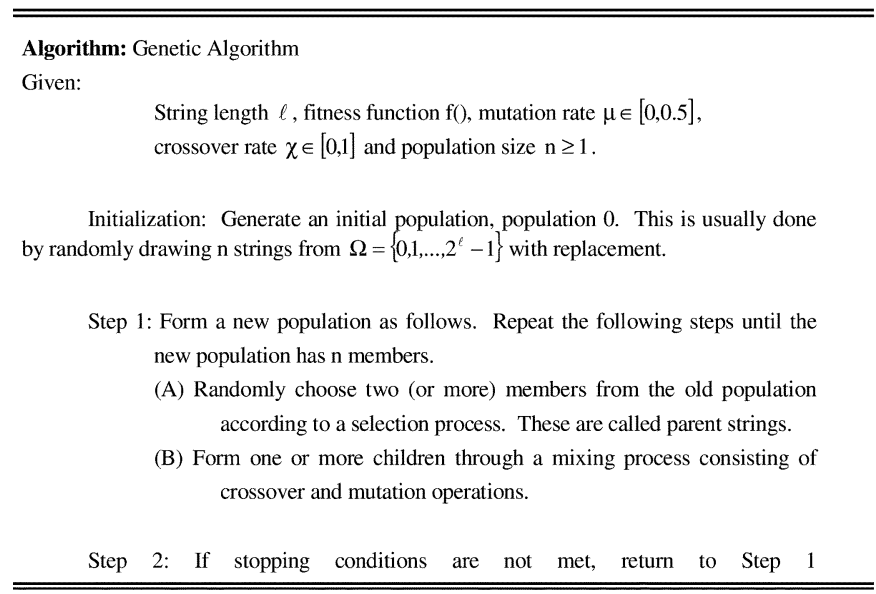

greater chance of being stochastically selected for the next generation. Second, selected strings are recombined, or crossed, in the hope of discovering better or fitter strings by combining genetic material. Third, the selected strings are randomly mutated to replace any lost diversity after selection and crossover. As such, GAs are a stochastic search technique. The mechanics of the GA are illustrated in Table I below.

Selection occurs similar to that of asexual reproduction in the natural world. Chromosomes that are deemed "fit" by measure of a predefined fitness function are stochastically more likely to be represented in future populations. The version of selection used for this model is rank selection. Rank selection starts by sorting the population according to fitness value. Each string receives new copies that are placed in the new population according to a function of this ordering. Several other variations of selection are discussed in [12].

Crossover implements a mating strategy for the combination of "good" genetic material between fit parents. After the selection procedure is complete, crossover is applied with a predetermined, fixed probability, called the crossover rate, usually ranging from 0.6 to 1.0. Uniform crossover is implemented in this model. Each corresponding bit pair between the two strings is exchanged with probability $2^{-\ell}$ [13], where $\ell$ is the length of the string. Other crossover schemes exist and are discussed in [12].

Mutation is the last operation on the population before the next generation is completely formed. In the binary case, muta- 
tion simply requires the mutated bit becomes its complement (i.e., 0 becomes 1 and vice-versa). Under uniform mutation, mutation is applied with a fixed, predetermined probability to each gene (each bit) in every string. The mutation rate is kept very low, usually between 0.001 and 0.005 , in order to keep the search from diversifying too rapidly. Other mutation schemes are available, see [12].

Nix and Vose in [14] developed a Markov chain (MC) model for genetic algorithms. Each state of the Markov chain represents a population of the GA. This model provides an exact representation for the expected populations of a GA over time. Using this model, one can compute maximum-likelihood estimates of crossover and mutation rates from existing data, rather than repeatedly executing the GA with various crossover/mutation rate combinations and attempting to find the best fit.

\section{EVOLUTIONARY MODEL FOR GROUP DECISION SUPPORT SYSTEMS}

The idea that groups undergo change and that the initial ideas or proposals submitted during a GDSS session are subject to adaptation is not a new idea. However, little has been done to incorporate the adaptation of potential solutions into an analytical model for GDSS. By using an evolutionary computing technique, for which an exact analytical model is available, such as the GA described above, we can begin to more accurately describe the search processes that occur during GDSS use. By search processes we include the generation of solutions as occurs during brainstorming activities, the exchange of information as occurs during negotiation activities as well as any refinement or learning of solutions that also may occur. For this particular study, we assume a simplified GDSS session, involving only the following. Provided a task (for example a resource allocation problem involving different and potentially conflicting constraints, costs and benefits to specific group members and the departments they represent), the group members will submit ideas or potential solutions to the group using the GDSS. This activity is analogous to the selection operation of the genetic algorithm. These ideas are exchanged between group members and extended or improved upon by other group members. This activity is analogous to the crossover operation of the genetic algorithm. In addition, the occasional random thought or idea is inserted into the collection of ideas. This action is similar to the mutation operation of the genetic algorithm. This process continues until the group agrees upon a solution or suspends the session for continuation at a later date.

We propose that group problem solving, when supported by GDSS, can be modeled by a genetic algorithm, utilizing selection, crossover and mutation. Each collection of proposed ideas or solutions generated by the group can be represented by a population of strings; each string in the population at time step t represents a current proposed solution at time t. Selection, crossover and mutation operate as above. As the generations evolve, the genetic algorithm should find either the best or at least a very good solution. We believe that GDSS supported groups can be viewed as generating and evolving solutions using a mechanism that can be modeled in this way.

We have chosen a genetic algorithm to model GDSS for several reasons. First, GAs are adaptive, meaning there is change over time, in response to the environment, including the fitness function and other constraints. The second reason for using a GA is a body of formal, mathematical theory has been developed to describe the expected behavior of the simple genetic algorithm. If groups using GDSS for particular tasks can be modeled as a GA, this theory could provide numerous insights into the group decision-making process. For example, variables and different environmental pressures thought to influence the process could be related to GA parameters and then factors such as the expected behavior of the system could be determined or optimized. Eventually, the model could be used as the basis for simulation studies, allowing researchers to examine more combinations of GDSS variables before conducting lab and field experiments, with the goal of better understanding different variables, the interactions between those variables, and the impacts upon GDSS decision quality.

\section{RESEARCH QUESTIONS}

The underlying assertion for this work is that groups using GDSS act like GAs using selection, crossover, and mutation. This assertion was initially tested in [5] using a simpler form of the GA. The enhanced GA will be tested in the first hypothesis. In the following, $\chi$ stands for "crossover rate" and $\mu$ stands for "mutation rate." Our main assertion is the following.

Assertion: Groups Using GDSS Act Like a Genetic Algorithm: We implement this GA using rank selection, uniform crossover, and uniform mutation as described above. We will specifically test this assertion by comparing the maximum likelihood ratios of the path probabilities of the estimated parameters $\left(\chi^{*}\right.$ and $\left.\mu^{*}\right)$ to the probability of these paths under a random search. This comparison is tested in the following hypothesis:

$H 1-\mathrm{P}_{\mathrm{i}, \mathrm{j}}\left(\chi^{*}, \mu^{*}\right)-\mathrm{P}_{\mathrm{i}, \mathrm{j}}(0.0,0.5) \neq 0$ : The above hypothesis will be tested using the methodology described in Section V below.

1) GDSS Variable Hypotheses: One of the issues facing group work is the social and political forces affecting members of the group, possibly affecting the quality of the group's decision-making. We hypothesize that proximate or face-to-face (FTF) groups respond more to group or "societal" pressures and tend to conform. This would lead to similar thought processes being explored in depth, rather than many different (and possibly conflicting) ideas being presented for consideration. Therefore, FTF groups tend to be more focused. Geographically dispersed or computer-mediated-communication (CMC) groups are exposed to fewer visual cues meaning a greater sense of anonymity, which could lead to the proposal of possibly very different solutions [3]. Research performed on distributed groups versus proximate groups has found that distributed groups exhibit greater degrees of depersonalization and impulsiveness, lowered inhibition, and generate "... more extreme opinions" [15, p. 328], [8]. In other words, CMC groups can be considered more explorative of the solution space than FTF groups. Under these assumptions of social and political motivations, FTF groups would be less likely to present completely new solutions than CMC groups. CMC group members would be more likely to explore alternate but possibly unpopular or politically less favorable solutions. These ideas lead to our next hypotheses. 
Hypothesis $H 2 a-\mu_{\mathrm{FTF}}-\mu_{\mathrm{CMC}}<0$ : We test H2a by comparing the maximum likelihood estimate of the mutation rates of both groups. If $\mathrm{H} 2 \mathrm{a}$ is true, the best fit mutation rate $\mu$ should be lower for FTF groups than CMC groups. In both FTF and CMC groups from Barkhi's study, there were three to four participants in each group, for a total of 48 groups.

Solution diversity within a population provides insight into the variation among the different solutions proposed by group members. As stated above, FTF groups are likely to conduct a less explorative search than CMC groups. Therefore, as the same number of group members searches less of the solution space in FTF groups than CMC groups, we would expect the diversity levels to average lower than CMC groups.

Diversity $(\Delta)$ can be measured as the average distance between the solutions, or points, in the solution space. GA research has concerned itself extensively with the concept of Hamming distance [12]. Mitchell in [16] defines Hamming distance as the number of locations or genes at which the corresponding values or bits differ. Other such distance measures are possible, however, Hamming distance represents the simplest and most widely used distance measure for complex search spaces in GA literature. We define $\Delta$ as

$$
\Delta=\frac{\sum_{i=1}^{n_{p}} d_{i}}{n_{p}}
$$

where $d_{i}$ is the Hamming distance between each pair $i$ and $n_{p}$ is the number of pairs.

Hypothesis $H 2 b-\Delta_{\mathrm{FTF}}-\Delta_{\mathrm{CMC}}<0$ : As $\Delta$ becomes small, $\chi$ should also become small for FTF groups. As there is less diversity within the group, the crossover rate, or the rate at which "parts" of proposals or ideas are exchanged, should become small, as most of the proposals are already identical. Correspondingly, $\Delta$ and $\chi$ should both be larger for CMC groups.

Hypothesis $H 2 c-\chi_{\mathrm{FTF}}-\chi_{\mathrm{CMC}}<0$ : We test this hypothesis by comparing the maximum likelihood estimates of the crossover rates $\chi$, between FTF and CMC groups. Given that diversity is lower for FTF groups as proposed in H2b, the estimated crossover rate $\chi$ should be lower for FTF groups than for CMC groups.

The next set of variable hypotheses examines the link between GA parameters and incentive structure. Often in GDSS research, group member incentive or payoff is assumed to be implicit (i.e., a normal part of the job). Very few studies have examined the impact of incentive on GDSS outcome.

Barkhi in [17] examined the effects of two different explicit incentive structures on a particular negotiation task. The task in their study was a type of resource allocation problem involving conflict, negotiation, and information asymmetry. Group members were provided cost and revenue data specific to the particular department of the member. The task was to select the set of customer orders that would maximize profit for the company given capacity and resource constraints. Group members received rewards that were tied to the profitability of either the firm as a whole (global incentive structure) or based on how well the individual departments controlled costs relative to revenues (local incentive structure). These two incentive structures studied are representative of the many possible permutations of the incentive variable.
That particular study reported that a local incentive structure resulted in greater organizational profit than did global incentive structure. They theorized that local group members, assuming rationality, could easily compute the marginal increase in reward as effort level increased and would select the best effort level for each customer order. Groups under global incentive receive increasing benefits as the group members' productivity increases. If the group members are unable to see the relationship between individual effort and group productivity, a tendency to "free ride" results as described in [17] and [18] effectively lowers group output. Groups under global incentive also tend to engage in gaming behavior as described in [17], reducing profit for the entire group. As groups engage in such behavior, the level of cooperation is often reduced in the face of uncertainty. Since there is little pressure on the global incentive group member to find the best solution possible, there would be little motivation to actively cooperate and quickly converge. Conversely, we expect local incentive group members to engage in more cooperative behavior in order to reach convergence. Therefore, we would expect the level of exploration in solutions proposed to be lower in local incentive groups and higher in global incentive groups (due to the increased competition).

Hypothesis $H 3 a-\mu_{\mathrm{L}}-\mu_{\mathrm{G}}<0$ : This hypothesis will be tested by comparing the maximum likelihood estimates of the mutation rates of both groups. The estimated mutation rate, $\mu$, should be lower for local (L) incentive groups than global (G) incentive groups.

Similarly, as the same number of group members are conducting a less explorative search in local incentive groups than for global incentive groups, the solutions should not vary as much in local incentive groups than in global incentive groups. We, therefore, believe the average level of diversity $(\Delta)$ as measured by the average Hamming distance will be lower for local incentive groups than for global incentive groups.

Hypothesis H3b- $\Delta_{\mathrm{L}}-\Delta_{\mathrm{G}}<0$ : We will test this hypothesis by comparing $\Delta$ for both global incentive and local incentive groups. $\Delta$ should be smaller for local incentive groups than for global incentive groups. As $\Delta$ becomes small, $\chi$ should also become small for local incentive groups. As there is less diversity within the group, the rate at which segments of solutions are exchanged will appear smaller, as most of the solutions are already identical.

Hypothesis $H 3 c-\chi_{\mathrm{L}}-\chi_{\mathrm{G}}<0$ : We will test this hypothesis by comparing the maximum likelihood estimates of the crossover rates $\chi$ between local and global incentive groups. Given that diversity is lower for local incentive groups as proposed in $\mathrm{H} 3 \mathrm{~b}$, the estimated crossover rate $\chi$ should be lower for local incentive groups than for global incentive groups.

\section{EXPERIMENTAL DATA}

To test and validate our model, we use data provided by Barkhi in [17]. We now present some of the details of these experiments, as the research variables used in Barkhi's study influenced the way in which we have related GA parameters to GDSS parameters. Barkhi's experiments examined the effects of various factors on the outcome of GDSS problem-solving tasks. He considered a mixed-motive task by which group members had to coordinate the final solution in the face of 
conflicting pay-off information. He constructed a group where each member represented a different department within a simulated manufacturing environment, the departments being labeled as production, purchasing, and marketing. Some of the groups in the study were comprised of the three members labeled as above and the others were comprised of four members (the previous three plus a designated "leader" who had override power on all decisions made within the group). There were 48 groups in his study. The group was assigned a combinatorial problem with a calculated payoff for each member.

Included in the research variables studied were proximity (face-to-face versus geographically distributed) and member incentive structure (local versus global). Barkhi tested two different incentive schemes. One scheme, local incentive, rewarded each manager based on how well the manager controlled actual costs compared to projected costs. The other scheme, global incentive, rewarded each manager based on an equal percentage of the organizational bonus, corresponding to organizational profit.

\section{A. Model Details}

This section presents the problem-specific details of the research model. The encoding of the strings, population sizing, fitness function, and GA operator implementation are described.

Each string in the population represents a set of orders to be filled as proposed by a manager or the leader in a specific generation. Each string is composed of 20 binary digits, each representing the inclusion (or exclusion) of a customer order by a one (or zero). A population consists of a number of solutions. The number varies from episode to episode, but there is no "natural" demarcation for groups using GDSS. GDSS supported groups can be modeled as having a dynamic population size. We propose the following scheme for modeling the populations.

The population-sizing scheme treats each group member (each functional area manager and leader, if present) as having ideas of nearly equal weight. Whenever a solution from a different manager is presented, the current generation is closed. For example, the marketing manager proposes a solution, then another solution immediately afterwards. The production manager then proposes a solution. This marks the end of the generation and the population size is three. For groups with a leader, the leader is simply regarded as another functional manager. However, several issues are raised. In the case where the GDSS participants are not anonymous, if a leader is present, there is the belief that leaders may exert a different type of influence over the decision-making process than do peers. Therefore, some account of this variance in influence should be taken in forming the generations. An example of an application of the sizing scheme is provided in Fig. 2. In this example, managers each contribute possible solutions to the discussion. These ideas are encoded into binary strings. For example, the marketing manager $(\mathrm{M})$ proposes a solution, followed by a solution from the production manager $(\mathrm{P})$, followed by another solution from the marketing manager $(\mathrm{M})$, followed by the leader of the group (L) and so forth. These proposed solutions are then temporally placed into populations. In Fig. 3, the ideas proposed in Fig. 2 are grouped into populations where each population has two strings $(n=2)$.

\begin{tabular}{|l|}
\hline \multicolumn{1}{|c|}{ Proposed Solution Sequence } \\
\hline M: 00100011010001000011 \\
P: 10100010010010001011 \\
M: 00010011010000000011 \\
L: 00100011010000000011 \\
L: 00100011010001000011 \\
P: 00100111010010000000 \\
P: 00100011010001000011 \\
\hline
\end{tabular}

Fig. 2. Population sizing scheme example: idea generation process.

\begin{tabular}{|l|}
\hline \multicolumn{1}{|c|}{ Populations } \\
\hline Population 0: \\
00100011010001000011 \\
10100010010010001011 \\
\hline Population 1: \\
00010011010000000011 \\
00100011010000000011 \\
\hline Population 2: \\
00100011010001000011 \\
00100111010010000000 \\
\hline Population 3: \\
00100011010001000011 \\
\hline
\end{tabular}

Fig. 3. Population sizing scheme example: generations formed from proposed solution sequence.

The fitness functions can be viewed as an implementation of the social welfare functions described in economic agency theory literature. Luce and Raiffa define social welfare function as "... a rule which associates to each profile of preference orderings (i.e., to each element of $\Re^{(n)}$ ) a preference ordering for society itself" [19, p. 332]. More specifically, the fitness function used is to be an additive utilitarian social welfare function as described in [20].

The GA as presented in [13] utilizes three operators: selection, crossover, and mutation. There exist several variations on each operator, for example, single-point versus uniform crossover [13]. An earlier version of our model implemented perhaps the simplest version of a GA as possible [5]. To better capture the nature of the GDSS search process, the model was enhanced from roulette wheel selection to rank selection and from single-point crossover to uniform crossover. Uniform crossover applied to two ideas-A and B-is illustrated in Fig. 4, where the crossed digits are highlighted in bold. Finally, uniform mutation is illustrated in Fig. 5, where the underlined bit is the one that is mutated.

As GDSS supported groups can be modeled as a GA as stated above, rank selection serves as a likely means of selection of the next $(t+1)$ generation. Rank selection is an intuitive means of selecting solutions that will serve as parent solutions for future generations and is described in [12] and [13]. Crossover is implemented in our model as uniform crossover as detailed in [12] and formally in [13]. Uniform crossover appears a likely candidate due to its ability to "mix and match" portions of the two parent solutions, rather than the one cut mechanism of singlepoint crossover. Mutation is implemented as uniform mutation also as described in [12] and [13]. 


\begin{tabular}{|c|}
\hline Before crossover \\
\hline Idea A: 100100111010010010 \\
\hline Idea B: 010010001001010010 \\
\hline \\
\hline After crossover \\
\hline Idea A': 010010101000010010 \\
\hline Idea B': 100100011011010010 \\
\hline
\end{tabular}

Fig. 4. Uniform crossover implementation.

\begin{tabular}{|c|}
\hline Before mutation \\
\hline Idea A':100100111001010010 \\
\hline \\
\hline After mutation \\
\hline Idea A"': 101100111001010010 \\
\hline
\end{tabular}

Fig. 5. Uniform mutation implementation where _ denotes the mutated bit.

TABLE II

Steps IN THE WilcoXon Matched-PAIRS Signed-RANKS TeST

1) Calculate the difference between each pair $D_{i}=x_{i 1}-x_{i 2}$ for $i=1, \ldots, n$ where $x_{i 1}$ and $x_{i 2}$ are the matched observations.

2) Assign ranks by placing each pair, $\left|\mathrm{D}_{\mathrm{i}}\right|$ in order and

record signs.

3) $\mathrm{T}^{+}=\sum_{\mathrm{i}=1}^{\mathrm{n}} \mathrm{D}_{\mathrm{i}}$ for $\mathrm{D}_{\mathrm{i}}>0$

4) Test statistic, $w_{l-\infty}$ is set for the two-tailed test at $\alpha=0.05$

\section{B. Methodology}

Several researchers have examined the evolutionary characteristics of systems by creating a simulation environment where a GA is used to mimic the behavior of some agent or group of agents. This research differs in that rather than simulate the groups used in Barkhi's study, we used the historical data from Barkhi to find a best-fit GA. We used the model proposed in [13] which gives transition probabilities to determine a likelihood function for the probabilities of each group's particular path through the solution space. Specifically, each population of a GA can be represented as a state in a Markov chain. The likelihood of moving from generation to generation can be expressed as a function of the selection and mixing operators [13]. The expected path probability, or likelihood, for a given Markov chain is

$$
\mathrm{P}_{\mathrm{j}_{1} \mathrm{j}_{2}}\left(\mathrm{~N}_{1}, \mathrm{~N}_{2}\right) \mathrm{P}_{\mathrm{j}_{2} \mathrm{j}_{3}}\left(\mathrm{~N}_{2}, \mathrm{~N}_{3}\right) \ldots \mathrm{P}_{\mathrm{j}_{\mathrm{T}-1} \mathrm{j}_{\mathrm{T}}}\left(\mathrm{N}_{\mathrm{T}-1}, \mathrm{~N}_{\mathrm{T}}\right)
$$

where $\mathrm{N}_{1}, \mathrm{~N}_{2}, \ldots, \mathrm{N}_{\mathrm{T}}$ are the population sizes at times $\mathrm{t}=$ $1,2, \ldots, \mathrm{T}$. We maximized this quantity over $\chi$ and $\mu$. Therefore, the maximum-likelihood function over $\chi$ and $\mu$ is

$$
\max _{\chi, \mu} \prod_{i=1}^{\mathrm{T}-1} \mathrm{P}_{\mathrm{j}_{\mathrm{i}}, \mathrm{j}_{\mathrm{i}+1}}\left(\mathrm{~N}_{\mathrm{i}}, \mathrm{N}_{\mathrm{i}+1}\right) .
$$

The maximum-likelihood estimates (MLEs) for these paths were calculated over all possible values of mutation $(0,0.5)$ and crossover $(0,1.0)$ with three-digit precision. Therefore, we estimated a best-fit mutation and crossover rate for each group,
TABLE III

TEST RESULTS FOR MAIN HYPOTHESIS

\begin{tabular}{lll}
\hline Hypothesis & Critical Value & Results \\
\hline H1 & $\mathrm{T}=1081$ & $(\mathrm{p}<.001)$ \\
& $\mathrm{w}_{.95}=691.1$ & Do Not Reject \\
\hline
\end{tabular}

assuming each group acted like a GA. This process is also known as learning the parameters of the Hidden Markov Model (HMM) within the data and was demonstrated in [7]. The best-fit path probability estimates for each group were compared with the path probability for a GA using random search, namely a GA where $\chi=0.0$ and $\mu=0.5$. If the groups behave significantly different than a GA using random search, then there is support for the hypothesis that the groups behave as a GA with specific GA operators and parameter values. We test this hypothesis by using the Wilcoxon matched-pairs signed-ranks test [21] described in Table II. The assumptions of the test are that the difference between each pair is a continuous random variable, the distribution of each difference is symmetric, the differences are mutually independent, the differences all have the same median, and the measurement scale is interval [22]. Each GDSS variable/GA parameter hypothesis is tested by means of a one-tailed $t$ test on the average parameter values, assuming unequal variances, at the $\alpha=0.05$ level to test for differences in the described groups.

\section{RESULTS}

The results of the test of Hypothesis H1, that groups using GDSS behave like a GA, are provided in the table below (Table III). The results indicate that there is strong evidence against groups using GDSS acting like a random search. At the 5\% significance level, these results specifically show that the GDSS groups examined did not have best-fit estimated crossover rates equal to 0.0 or best-fit estimated mutation rates equal to 0.5 . Therefore, we have evidence that the GDSS groups are conducting a search process consistent with the search undertaken by a GA.

Our first parameter hypothesis, H2a, stated that FTF groups are likely to have less radical or extreme search than CMC groups. In order to test this hypothesis, we compared the best-fit mutation rate $\mu$ between FTF and CMC groups. For this hypothesis to not be rejected, FTF groups must have a smaller $\mu$ than CMC groups. This hypothesis was rejected at $\alpha=0.05$. The overall mean for FTF groups is slightly higher $(0.023)$ than the overall mean for CMC groups (0.022) but not significantly so $(p=0.44)$. Therefore, we conclude there is no significant difference in the level of radicality of the search between FTF and CMC groups. These results are presented in Table IV.

The next hypothesis posed compares the level of diversity (difference in solutions as measured by the Hamming distance) between FTF and CMC groups. Hypothesis H2b stated FTF groups are likely to have a lower diversity value (as measured by $\Delta$ ) than CMC groups. This hypothesis was rejected at $\alpha=0.05$. The difference in the means for the two groups is significant; however, the CMC groups have the lower level of diversity than the FTF groups. Therefore, we conclude FTF groups do not have a lower diversity value than CMC groups. The one-tailed t test results for the average diversity level are presented in Table IV. 
TABLE IV

REsults of Statistical TESTS ON COMMUNICATIONS CHANNEL HyPOTHESES

\begin{tabular}{llll}
\hline Hypothesis & $\begin{array}{l}\text { FTF } \\
\text { Mean }\end{array}$ & $\begin{array}{l}\text { CMC } \\
\text { Mean }\end{array}$ & Results \\
\hline $\mathrm{H} 2 \mathrm{a}$ & 0.023 & 0.022 & $\begin{array}{l}\mathrm{p}=0.44) \\
\text { Reject H2a }\end{array}$ \\
\hline $\mathrm{H} 2 \mathrm{~b}$ & 1.277 & 0.860 & $\begin{array}{l}(\mathrm{p}=0.04) \\
\text { Reject H2b }\end{array}$ \\
\hline $\mathrm{H} 2 \mathrm{c}$ & 0.100 & 0.206 & $(\mathrm{p}=0.10)$ \\
& & & $\begin{array}{l}\text { Do Not Reject } \\
\text { H2c }\end{array}$ \\
\hline
\end{tabular}

TABLE V

REsults of Statistical Tests on InCENTIVE Structure

\begin{tabular}{llll}
\hline Hypothesis & $\begin{array}{l}\text { Local } \\
\text { Mean }\end{array}$ & $\begin{array}{l}\text { Global } \\
\text { Mean }\end{array}$ & Results \\
\hline $\mathrm{H} 3 \mathrm{a}$ & 0.017 & 0.028 & $\begin{array}{l}(\mathrm{p}=0.05) \\
\text { Do not reject } \\
\text { H3a }\end{array}$ \\
\hline $\mathrm{H} 3 \mathrm{~b}$ & 1.001 & 1.094 & $\begin{array}{l}(\mathrm{p}=0.33) \\
\text { Reject H3b }\end{array}$ \\
\hline $\mathrm{H} 3 \mathrm{c}$ & 0.199 & 0.120 & $(\mathrm{p}=0.17)$ \\
& & & Reject H3c \\
\hline
\end{tabular}

Our final hypothesis comparing FTF groups with CMC groups relates the crossover rate $\chi$ to the diversity rate. Hypothesis $\mathrm{H} 2 \mathrm{c}$ stated if the diversity $\Delta$ is close to zero, FTF groups are likely to behave like a GA having a lower crossover rate $\chi$ than $\mathrm{CMC}$ groups. We find that there is some support for $\mathrm{H} 3 \mathrm{a}$, although it is not strongly significant $(\mathrm{p}-$ value $=0.10)$. The one-tailed $t$ test results for the best-fit estimated crossover rates are presented in Table IV.

Hypothesis H3a stated that groups having a local incentive structure should have a lower mutation rate than groups having a global incentive structure. We do not reject H3a at $\alpha=0.05$. This finding supports the hypothesis that local incentive groups appear to converge to a solution faster than global incentive groups. These results are reported in Table $\mathrm{V}$ below.

Hypothesis H3b stated groups having a local incentive structure should have a lower diversity $\Delta$ than global incentive structure. This hypothesis was rejected at the $\alpha=0.05$ level. However, we found that local incentive groups have a lower $\Delta(1.001)$ than global incentive groups (1.094).

The crossover rate $\chi$ for global and local incentive groups was also compared. Hypothesis $\mathrm{H} 3 \mathrm{c}$ stated local incentive groups are likely to experience a lower estimated crossover rate than global incentive groups. Again, we reject this hypothesis at $\alpha=0.05$. However, it is noted that local incentive groups have a lower estimated crossover rate $\chi(0.199)$ than global incentive groups (0.120), providing limited support for this hypothesis.

\section{CONCLUSION}

As discussed in the previous section, we conclude that groups using GDSS do act like a GA using selection, crossover, and mutation. Also in this research, we studied two parameters: the crossover and mutation rates, $\chi$ and $\mu$, respectively, and their effects on communications channel and incentive structure. We experienced mixed success in linking the GA parameters of $\mu$ and $\chi$ to the particular GDSS variables examined in Barkhi's study [17]. The linkage between the parameters $\chi, \mu$ and the diversity level $\Delta$ and communications channel was tenuous. There appeared to be no difference in estimated mutation rates between the two communications channels. There was a significant difference in diversity levels between the two channels but not in the direction hypothesized. There was a weakly significant difference between the estimated crossover rates of the two channels, implying that there are measurable differences between the search processes of the two experimental groups. It is clear that more experimental data are required for fully understanding the phenomena involved in this particular GDSS model.

The linkages between $\chi, \mu$ and $\Delta$ were more interesting for the incentive structure variable. A significant difference in estimated mutation rates was observed between the local incentive groups and the global incentive groups. Although not significant, the average diversity levels and estimated crossover rates were also different, suggesting fundamental differences in the search processes of the two experimental groups.

It is readily apparent that more data sets are required to carry out robust and accurate testing of this particular model, since most results came out as anticipated but were not always statistically significant. Additionally, the relationships between other GDSS variables, such as group size, composition, task type, anonymity, and others, and GA parameters need to be examined, including any interaction effects, for a more complete analysis. However, it is clear that the experimental GDSS groups did not perform a random search, but in fact conducted a search consistent with a genetic search with identifiable crossover and mutation rates. It is also weakly confirmed that a relationship exists between incentive structure and GA parameters. The possible relationship between communication channel and GA parameters requires further analysis. One very positive feature of the approach demonstrated in this research is that the GA approach itself appears robust in the general case as shown in [7].

One implication of this finding is that work may begin on designing useful and more realistic simulation models for GDSS experiments. Different experimental configurations might be examined before conducting time-consuming and expensive experiments with actual groups. Additionally, the underlying Markov Chain model of the GA can be utilized to study the expected behavior of the system and hopefully gain further insights into GDSS processes.

This project provides many opportunities for future research. The GDSS model could be improved by incorporating the more expressive GA operators described in the previous section. Features of other models could be included, particularly the economic model for GDSS as proposed in [9]. Obviously, more data from actual GDSS experiments is required to further validate this model, especially from experiments that further examine the variables studied in [17].

There are also future research implications of particular interest to GDSS researchers. Not only are there other GDSS 
configurations of interest, but also location and time-dependent meetings, meetings that occur over an extended period of time, and meetings that take place within virtual organizations [23]. Other task types and GDSS variables, such as group size, composition, anonymity, and incentive structures can be examined within the context of the model. This model can eventually be applied to non-GDSS-supported groups, contributing to the understanding of all group interactions.

\section{REFERENCES}

[1] H. A. Simon, The New Science of Management Decision. Englewood Cliffs, NJ: Prentice-Hall, 1977.

[2] G. DeSanctis and R. B. Gallupe, "A foundation for the study of group decision support systems," Manage. Sci., vol. 33, no. 5, pp. 589-609, 1987.

[3] J. F. Nunamaker, A. R. Dennis, J. S. Valacich, D. R. Vogel, and J. F. George, "Electronic meeting systems to support group work," Commun. ACM, vol. 34, no. 7, pp. 40-61, 1991.

[4] R. Hirokawa and D. Johnson, "Toward a general theory of group decision making development of an integrated model," Small Group Behavior, vol. 20 , no. 4 , pp. 500-523, 1989.

[5] J. Rees and G. J. Koehler, "Evolution in groups: A genetic algorithm approach to group decision support systems," Inform. Technol. Manage., vol. 3 , no. 3, 2002

[6] - "Leadership and group search in group decision support systems," Decision Support Syst., vol. 30, no. 1, 2000.

[7] — Learning Genetic Algorithm Parameters Using Hidden Markov Chains, 2003. submitted for publication.

[8] J. S. Valacich and A. R. Dennis, "A mathematical model of performance of computer-mediated groups during idea generation," J. Manage. Inf. Syst., vol. 11, no. 1, pp. 59-72, 1994

[9] B. Gavish and J. Kalvenes, "Economic issues in group decision support systems," in Proc. 1st INFORMS Conf. Inform. Syst. Technol., H. Pirkul and M. Shaw, Eds. Washington, DC: INFORMS, 1996, pp. 18-27.

[10] B. Gavish, J. Gerdes Jr., and S. Sridhar, " $\mathrm{CM}^{3}$, looking into the third and fourth dimensions of GDSS," in Information and Collaboration Models of Integration, S. Y. Nof, Ed. Norwell, MA: Kluwer, 1994, pp. 269-299.

[11] — " $\mathrm{CM}^{3}$ —A distributed group decision support system," IIE Trans., vol. 27, no. 6, pp. 722-733, 1995.

[12] D. E. Goldberg, Genetic Algorithms in Search, Optimization, and Machine Learning. Reading, MA: Addison-Wesley, 1989.

[13] M. D. Vose, The Simple Genetic Algorithm: Foundations and Theory. Cambridge, MA: MIT Press, 1999.

[14] M. A. Nix and M. D. Vose, "Modeling genetic algorithms with Markov chains," Annals of mathematics and artificial intelligence, vol. 5, no. 1, pp. 79-88, 1992

[15] C. L. Sia, B. C. Y. Tan, and K.-K. Wei, "Will distributed GSS groups make more extreme decisions? An empirical study," in Proc. 17th Int. Conf. Inform. Syst., J. I. DeGross, S. Jarvenpaa, and A. Srinivasan, Eds., Cleveland, OH, 1996, pp. 326-338.

[16] M. Mitchell, An Introduction to Genetic Algorithms. Cambridge, MA: The MIT Press, 1996.
[17] R. Barkhi, "An empirical study of the impact of proximity, leader and incentives on negotiation process and outcomes in a group decision support setting," Ph.D. dissertation, The Ohio State Univ., Columbus, OH, 1995

[18] M. Diehl and W. Stroebe, "Productivity loss in brainstorming groups: Toward the solution of a riddle," J. Personality Social Psychology, vol. 53, no. 3, pp. 497-509, 1987.

[19] R. D. Luce and H. Raiffa, Games and Decisions Introduction and Critical Survey. New York: Dover, 1957.

[20] J. C. Moore, H. R. Rao, A. Whinston, K. Nam, and T. S. Raghu, "Information acquisition policies for resource allocation among multiple agents," Inf. Syst. Res., vol. 8, no. 2, pp. 151-170, 1997.

[21] S. Siegel, Nonparametric Statistics for the Behavioral Sciences. New York: McGraw-Hill, 1956

[22] W. J. Conover, Practical Nonparametric Statistics. New York: Wiley, 1971.

[23] S. L. Jarvenpaa and B. Ives, "The global network organization of the future: Information management opportunities and challenges," $J$. Manage. Inf. Syst., vol. 10, no. 4, pp. 25-57, 1994.

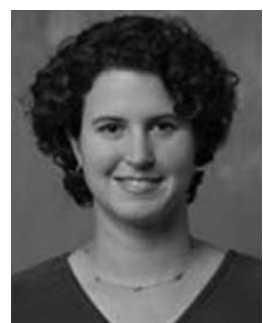

Jackie Rees received the B.S.B.A. and Ph.D. degrees in decision and information sciences from the University of Florida, Gainesville, in 1992 and 1998, respectively.

Currently, she is an Assistant Professor of Management Information Systems with the Krannert Graduate School of Management at Purdue University, West Lafayette, IN, where she has been since 1998. She was a Programmer Analyst from 1992 to 1994. Her current research interests include genetic algorithms and risk management for information security and privacy.

Prof. Rees is a member of the Association for Information Systems, INFORMS, and the Decision Sciences Institute.

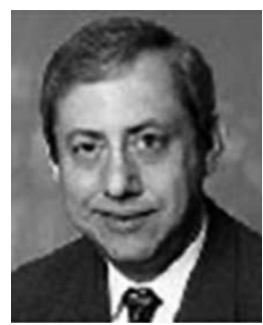

Gary J. Koehler received the B.S., M.S., and Ph.D. degrees in chemistry, physiology, and management science from Purdue University, West Lafayette, IN, in 1969,1970 , and 1973 , respectively.

Currently, he is the John B. Higdon Eminent Scholar of Management Information Systems in the Warrington College of Business at the University of Florida, Gainesville, where he has been since 1974. He was a Visiting Assistant Professor with faculty at Northwestern University, Evanston, IL, from 1973 to 1974 . His current research interests include GA theory and eCommerce-related areas.

Prof. Koehler is a member of the Association for Information Systems, INFORMS, the Decision Sciences Institute and the Association for Computing Machines. 